\title{
Egg-allergic patients gain greater access to H1N1 vaccine after completion of clinical trial
}

Published at www.cmaj.ca on Dec. 2, 2009

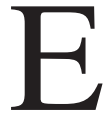
gg-allergic Quebecers have obtained greater access to the pandemic (H1N1) 2009 vaccine as a result of the first rapid clinical trial completed by Canada's new influenza research network, which hopes its work will extend access to similar patients across Canada.

The unpublished clinical trial, coordinated by Dr. Gaston De Serres, a medical epidemiologist at the Institut national de santé publique du Québec in Montréal involved work with four allergists in the province who administered the adjuvanted pandemic (H1N1) 2009 vaccine to 952 children and adults who are allergic to eggs. The trial was sponsored by the Public Health Agency of Canada-Canadian Institutes of Health Research Influenza Research Network.

The vaccinations, which took place in Quebec's four university hospitals, were completed by Nov. 11, 2009. Despite being allergic to eggs, none of the patients experienced anaphylactic reactions to the egg protein in the vaccine, De Serres says.

"Among these more than 900 patients, none had that," Serres says. "That's a reassuring result. There were two allergic reactions that were treated with [diphenhydramine hydrochloride] Benadryl but were mild cutaneous reactions. We were pretty happy with that."

As a result of the trial, De Serres and his colleagues recommended a change in the guidelines for vaccinating egg-allergic patients. Current guidelines recommend that these patients only be vaccinated in the presence of an allergist. But that is problematic during a mass vaccination campaign given the scant number of allergists to serve the $1 \%-2 \%$ of the population who are allergic to eggs.

De Serres' group recommended that allergists need not be present, as long as a physician is close by and the vac-

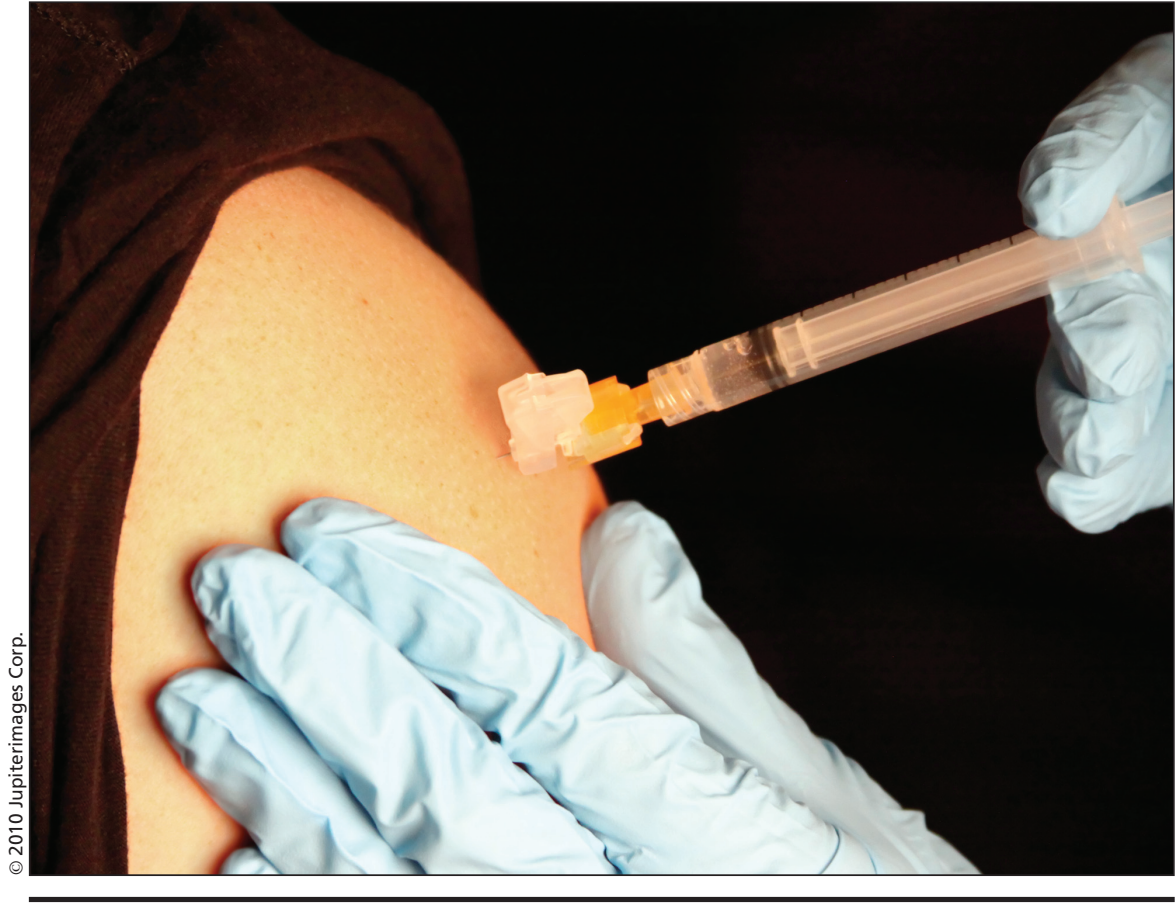

The Quebec Ministry of Health expanded vaccinations to egg-allergic patients as a result of clinical trial data indicating that subjects were not experiencing anaphylactic reactions to the egg protein in pandemic (H1N1) 2009 vaccine.

cine is administered in a hospital setting. The recommendation was adopted by the Association des allergologues et immunologues du Québec on Nov. 13, 2009. After approval from the Quebec Ministry of Health, expanded vaccinations to egg-allergic patients began occurring at hospitals during the week of Nov. 16, 2009.

In Quebec, nurses administering the vaccine to these patients have been provided with the same algorithm used in the clinical trial. It enables the health care professional to judge whether the patient needs a single dose or a divided dose. Patients judged to have a more severe risk of anaphylaxis, such as those with fever or respiratory problems, first receive $10 \%$ of the usual dose and the remaining $90 \%$ roughly 10 minutes later, provided they have not had a reaction to the first dose. Patients who receive a single dose must stay in the waiting room for 60 minutes instead of the usual 15, De Serres says.

The speed of the trial enabled the researchers to quickly translate their results into action, De Serres says. "We want to expand the access (to the vaccine) as long as it is safe."

The trial reassured allergists that vaccinations of egg-allergic patients could be delegated to other medical professionals, says Dr. Norman Dubé, president of Association des allergologues et immunologues du Québec. "We know by this study that the risk appears to be minimal - practically zero."

There are 4000-5000 children in Quebec who are allergic to eggs, and only 52 allergists serving the province. "So we were not able to vaccinate all of these patients under our immediate supervision, obviously," Dubé says. "It was necessary work that had to be done and we used this necessary task to 
collect the numbers. Hopefully that will lead us to reassure people at large about this kind of vaccine."

In total, 24 Canadians had experienced anaphylactic reactions to the pandemic vaccine as of Nov. 25, 2009, Dr. David Butler-Jones, Canada's chief public health officer, told a press conference.

De Serres has sent the results of the trial to the Canadian Society of Allergy and Immunology in hopes other provinces use the findings to expand vaccination programs for their eggallergic patients. The data from the trial is not yet available publicly and De Serres says they haven't determined how it will be released.

The new influenza research network involves 90 researchers from across Canada. It is conducting pandemic trials around five themes, including rapid trials, vaccine safety, vaccine effectiveness, measurement of vaccine uptake and assessment of vaccine delivery methods, says Dr. David
Scheifele, coprincipal investigator for rapid trials component of the network, and director of the vaccine evaluation centre at B.C. Children's Hospital.

There are four other ongoing rapid trials involving 1025 children and adults. One, involving 300 children aged 6-35 months, is assessing whether they need one or two doses of the adjuvanted vaccine for optimal protection.

A second study, involving 300 adults aged $20-59$, is assessing whether there's an adverse reaction in people who receive the adjuvanted pandemic vaccine along with the seasonal influenza vaccine.

A third study will recruit 200 Aboriginal adults and 75 Aboriginal children in Winnipeg, Manitob,a and Edmonton, Alberta, to compare their reactions to the adjuvanted vaccine with those of non-Aboriginal adults and children in the other trials. "The key question is whether the dosing recommendation for the general population is appropriate for Aboriginal children and adults as well," Scheifele says.

The final study involves $150 \mathrm{HIV}$ infected adults to see whether one or two doses of adjuvanted pandemic vaccine provide adequate protection.

Although the numbers enrolled in these trials are not large, "we'll add some important information to the story," Scheifele says.

Scheifele adds that data from half a dozen studies in different countries would be beneficial before making universal pronouncements about a vaccine's safety and effectiveness. But because the trials look at a realistic cross-section of Canadian children and adults, including Aboriginals, "I think our results will provide pretty good guidance for the Canadian situation," he says.

The network hopes to report its completed data by February. - Laura Eggertson, Ottawa, Ont.

DOI:10.1503/cmaj.109-3123 